\title{
Strict System Equivalence of 2D Linear Discrete State Space Models
}

\author{
Mohamed S. Boudellioua \\ Department of Mathematics and Statistics, Sultan Qaboos University, P.O. Box 36, Al-Khodh, 123 Muscat, Oman
}

Correspondence should be addressed to Mohamed S. Boudellioua, boudell@squ.edu.om

Received 10 October 2011; Revised 30 January 2012; Accepted 6 February 2012

Academic Editor: L. Z. Yu

Copyright (C) 2012 Mohamed S. Boudellioua. This is an open access article distributed under the Creative Commons Attribution License, which permits unrestricted use, distribution, and reproduction in any medium, provided the original work is properly cited.

The connection between the polynomial matrix descriptions (PMDs) of the well-known regular and singular 2D linear discrete state space models is considered. It is shown that the transformation of strict system equivalence in the sense of Fuhrmann provides the basis for this connection. The exact form of the transformation is established for both the regular and singular cases.

\section{Introduction}

Multidimensional linear systems theory has attracted many researchers in recent years. The reason is the wide application in areas such as signal processing, linear multipass processes, iterative learning control systems, and delay-differential systems. An area of interest has been the equivalence of systems. There are various notions of equivalence proposed in the literature, and the most basic concept of equivalence would require the preservation of the transfer function matrix of the system. For 1D systems, Rosenbrock [1] introduced the well-known definition of strict system equivalence, using a polynomial matrix description (PMD) setting. Subsequently, Rosenbrock's strict system equivalence was generalized by Fuhrmann [2] for PMDs not necessarily having the same size. A 2D version of Fuhrmann's definition of strict system equivalence has been proposed by Johnson [3] and Pugh et al. [4]. This transformation often referred to as zero coprime system equivalence or Fuhrmann's strict system equivalence (F-SSE) displays some interesting properties and has been shown by Pugh et al. [5] and Zerz [6] to provide the basis of a $n$-D generalization of Rosenbrock's least-order characterization. Recently Pugh et al. [7, 8] and Boudellioua [9] have shown that F-SSE forms the basis of the reduction of an arbitrary 2D PMD to various singular state space forms. Furthermore this transformation was shown to form the basis of a simplification method in linear multidimensional systems [10]. In this paper, it is shown that F-SSE provides the connection between the various $2 \mathrm{D}$ state space linear models proposed by Roesser [11], Attasi [12], FornasiniMarchesini [13], and Kaczorek [14].

\section{Equivalence of 2D PMDs}

Let $D=\mathbb{R}\left[z_{1}, z_{2}\right]$ denote the ring of polynomials in the indeterminates $z_{1}$ and $z_{2}$ with coefficients in a specified field of real numbers $\mathbb{R}$. The notion of polynomial matrix description was first introduced by Rosenbrock [1]. It arises from the polynomial system description

$$
\begin{gathered}
T x=U u, \\
y=V x+W u,
\end{gathered}
$$

where $x \in \mathbb{R}^{p}$ is the state vector, $u \in \mathbb{R}^{n}$ is the input vector, and $y \in \mathbb{R}^{m}$ is the output vector. T, $U, V$, and $W$ are polynomial matrices with elements in $D$ of dimensions $r \times p$, $r \times n, m \times p$, and $m \times n$, respectively. The system in (1) gives rise to the PMD

$$
P=\left(\begin{array}{cc}
T & U \\
-V & W
\end{array}\right)
$$


A matrix description (2) in which $T$ has full row rank and $V=H T$ for some rational matrix $H$ is called an admissible matrix description, and its transfer function is

$$
G=H U+W
$$

The following definitions are needed for the results of the paper.

Definition 1. Two polynomial matrices $P_{1}$ and $S_{1}$ of appropriate dimensions and with elements in $D$ are said to be zero left (right) coprime if the matrix

$$
\left(\begin{array}{ll}
P_{1} & S_{1}
\end{array}\right)\left(\left(\begin{array}{ll}
P_{1}^{T} & S_{1}^{T}
\end{array}\right)^{T}\right)
$$

admits a right inverse over $D$.

As in the $1 \mathrm{D}$ case, the zero structure of a multivariate polynomial matrix is a crucial indicator of the system behavior. Zerz [15] has shown that the controllability and observability of a system in the behavioral setting is connected to the zero structure of the associated polynomial matrix. The zero structure of a multivariate polynomial matrix is completely captured by the determinantal ideals as given by Zerz [16].

Definition 2. Let $P \in D^{p \times q}$, the ith-order determinantal ideal $\ell_{i}$ of $P$ be the polynomial ideal generated by the $i \times i$ minors of $P$.

The determinantal ideals $\ell_{i}$ of $P$ satisfy the following:

$$
\ell_{0} \supseteq \ell_{1} \supseteq \ell_{2} \supseteq \cdots \ell_{t} \text {, }
$$

where $t$ is the normal $\operatorname{rank}$ of $P$ and $\ell_{0}=D$.

Prior to introducing the concept of Fuhrmann's strict system equivalence in the $2 \mathrm{D}$ setting, the following transformation relating two polynomial matrices is noted.

Definition 3. Two polynomial matrices $P_{1}$ and $P_{2}$ with elements in $D$ are said to be zero coprime equivalent if they are related by

$$
S_{2} P_{1}=P_{2} S_{1}
$$

where $P_{1}, S_{1}$ are zero left coprime and $P_{2}, S_{2}$ are zero right coprime.

The transformation of zero coprime equivalence can be regarded as the classical unimodular equivalence coupled with a trivial expansion or deflation of the matrices. Despite its apparently restrictive form, this transformation displays some interesting properties. Johnson [3] and Pugh et al. $[4,17]$ have shown that zero coprime equivalence exhibits fundamental algebraic properties amongst its invariants as illustrated by the following.

Lemma 4 (Pugh et al. [17]). Suppose that two polynomial matrices $P$ and $Q \in \mathbb{P}(m, n)$ are related by zero coprime equivalence, and let $l_{j}^{[P]}$ for $j=1, \ldots, h=\min \left(r^{[P]}+m, r^{[P]}+n\right)$ denote the ideal generated by the $j \times j$ minors of $P$, and let $l_{i}^{[Q]}$ for $i=1, \ldots, k=\min \left(r^{[Q]}+m, r^{[Q]}+n\right)$ denote the ideal generated by the $i \times i$ minors of $Q$. Then

$$
\ell_{h-i}^{[P]}=\ell_{k-i}^{[Q]}, \quad i=0, \ldots, \bar{h},
$$

where $\bar{h}=\min (h-1, k-1)$ and $\forall i>h$,

$$
\ell_{h-i}^{[P]}=\langle 1\rangle \text {, }
$$

or

$$
\ell_{k-i}^{[Q]}=\langle 1\rangle
$$

in case $i<h$ or $i<k$.

In the context of PMDs a special case of the transformation given in (6) is used.

Definition 5. Let $\mathbb{P}(m, n)$ denote the class of $(r+m) \times(p+n)$ admissible PMDs where $m, n$, and $r+p$ are fixed positive integers. Two PMDs $P_{1}$ and $P_{2} \in \mathbb{P}(m, n)$ are said to be FSSE if they are related by the following:

$$
\underbrace{\left(\begin{array}{cc}
M & 0 \\
X & I_{m}
\end{array}\right)}_{S_{1}} \underbrace{\left(\begin{array}{cc}
T_{1} & U_{1} \\
-V_{1} & W_{1}
\end{array}\right)}_{P_{2}}=\underbrace{\left(\begin{array}{cc}
T_{2} & U_{2} \\
-V_{2} & W_{2}
\end{array}\right)}_{P_{1}} \underbrace{\left(\begin{array}{cc}
N & Y \\
0 & I_{n}
\end{array}\right)}_{S_{2}}
$$

where $P_{1}, S_{1}$ are zero left coprime, $P_{2}, S_{2}$ are zero right coprime, and $M, N, X$, and $Y$ are polynomial matrices over $D$ of appropriate dimensions.

Lemma 6 (Pugh et al. [17]). The transformation of F-SSE preserves the transfer function of $P$ and the determinantal ideals in the sense of Lemma 4 of the matrices

$$
T, P,\left(\begin{array}{ll}
T & U
\end{array}\right), \quad\left(\begin{array}{c}
T \\
-V
\end{array}\right) .
$$

\section{Equivalence of Regular 2D Linear Models}

State space models play an important role in the theory of 1D finite-dimensional linear systems. During the last decades extensions have been made of the state space representation to the more general 2D systems. One such extension is the discrete linear state space models proposed by Roesser [11], Attasi [12] and Fornasini-Marchesini [13]. It is well known that these models are not independent of each other and that the standard Roesser model is the most general in which the two other models can be embedded. In the following the exact polynomial connection between the various models is established.

The first 2D standard discrete linear model was introduced by Roesser [11] and can be described by the following equations:

$$
\begin{gathered}
\left(\begin{array}{l}
x_{1}(i+1, j) \\
x_{2}(i, j+1)
\end{array}\right)=\left(\begin{array}{ll}
A_{11} & A_{12} \\
A_{21} & A_{22}
\end{array}\right)\left(\begin{array}{l}
x_{1}(i, j) \\
x_{2}(i, j)
\end{array}\right)+\left(\begin{array}{l}
B_{1} \\
B_{2}
\end{array}\right) u(i, j), \\
y(i, j)=\left(\begin{array}{ll}
C_{1} & C_{2}
\end{array}\right)\left(\begin{array}{l}
x_{1}(i, j) \\
x_{2}(i, j)
\end{array}\right)+D u(i, j),
\end{gathered}
$$


where $x_{1}(i, j), x_{2}(i, j)$ are, respectively, the horizontal and vertical state vectors, $u(i, j)$ is the input vector, $y(i, j)$ is the output vector at cell $(i, j), A_{i j}, B_{i}, C_{i}$, and $D$ are constant real matrices of appropriate dimensions. Then, taking the $2 \mathrm{D} z$ transform of (12) and assuming zero boundary conditions yield the PMD:

$$
P_{\mathrm{R}}=\left(\begin{array}{cc|c}
z_{1} I-A_{11} & -A_{12} & B_{1} \\
-A_{21} & z_{2} I-A_{22} & B_{2} \\
\hline-C_{1} & -C_{2} & D
\end{array}\right) .
$$

The second 2D discrete linear model was introduced by Attasi [12]:

$$
\begin{aligned}
x(i+1, j+1)= & A_{2} x(i+1, j)+A_{1} x(i, j+1) \\
& -A_{1} A_{2} x(i, j)+B u(i, j), \\
y(i, j)= & C x(i, j),
\end{aligned}
$$

in which $A_{1}$ and $A_{2}$ are assumed to commute. The corresponding matrix description for this model is

$$
P_{A}=\left(\begin{array}{c|c}
z_{1} z_{2} I-z_{1} A_{2}-z_{2} A_{1}+A_{1} A_{2} & B \\
\hline-C & 0
\end{array}\right) .
$$

An alternative model was proposed by Fornasini and Marchesini [13] which is given by

$$
\begin{aligned}
x(i+1, j+1)= & A_{2} x(i+1, j)+A_{1} x(i, j+1) \\
& +A_{0} x(i, j)+B u(i, j), \\
y(i, j)= & C x(i, j) .
\end{aligned}
$$

This model is referred to as the first Fornasini-Marchesini model, and the corresponding matrix description is

$$
P_{\mathrm{FM} 1}=\left(\begin{array}{c|c}
z_{1} z_{2} I-z_{1} A_{2}-z_{2} A_{1}-A_{0} & B \\
\hline-C & 0
\end{array}\right) .
$$

Another model given by Fornasini and Marchesini [18] is the so-called second Fornasini-Marchesini model:

$$
\begin{aligned}
x(i+1, j+1)= & A_{2} x(i+1, j)+A_{1} x(i, j+1) \\
& +\bar{B}_{1} u(i, j+1)+\bar{B}_{2} u(i+1, j), \\
y(i, j)= & C x(i, j) .
\end{aligned}
$$

The corresponding system matrix is given by:

$$
P_{\mathrm{FM} 2}=\left(\begin{array}{c|c}
z_{1} z_{2} I-z_{1} A_{2}-z_{2} A_{1} & z_{1} \bar{B}_{2}+z_{2} \bar{B}_{1} \\
\hline-C & 0
\end{array}\right) .
$$

Because of the assumption on commutativity on $A_{1}$ and $A_{2}$, it has been shown by Morf et al. [19] that Attasi's model (14) is a special case of that of the first Fornasini-Marchesini model (16) and that the latter can be written in the form of Roesser's model (12) which itself is a special case of
Fornasini-Marchesini second model (18) upon assuming the following in (18):

$$
\begin{array}{cc}
A_{1}=\left(\begin{array}{cc}
A_{11} & A_{12} \\
0 & 0
\end{array}\right), \quad A_{2}=\left(\begin{array}{cc}
0 & 0 \\
A_{21} & A_{22}
\end{array}\right), \\
\bar{B}_{1}=\left(\begin{array}{c}
B_{1} \\
0
\end{array}\right), \quad \bar{B}_{2}=\left(\begin{array}{c}
0 \\
B_{2}
\end{array}\right) \\
C=\left(\begin{array}{ll}
C_{1} & C_{2}
\end{array}\right) .
\end{array}
$$

With these assumptions, the system matrix in (19) become:

$$
\text { PFM2s }=\left(\begin{array}{ccc}
z_{2}\left(z_{1} I-A_{11}\right) & -z_{2} A_{12} & z_{2} B_{1} \\
-z_{1} A_{21} & z_{1}\left(z_{2} I-A_{22}\right) & z_{1} B_{2} \\
\hline C_{1} & C_{2} & 0
\end{array}\right) .
$$

The PMD in (21) is clearly not F-SSE to the PMD in (13). However if a weaker transformation over the rational ring $\mathbb{R}\left(z_{1}, z_{2}\right)$ is used, the second Fornasini-Marchesini system matrix (21) can be clearly reduced to a Roesser's model (12) by simply cancelling the common factors $z_{2}$ and $z_{1}$ in the first and second block rows of PFM2s, respectively. In the following, the exact form of the connection between the PMDs (13) and (17) is established and shown to be F-SSE.

Theorem 7. Given an arbitrary Fornasini-Marchesini matrix description $P_{F M 1}$ in the form (17), then $P_{F M 1}$ is related to the Roesser matrix description $\bar{P}_{R}$ in the form (13) by the following F-SSE transformation:

$$
S_{1} P_{F M 1}=\bar{P}_{R} S_{2},
$$

where

$$
\begin{gathered}
S_{1}=\left(\begin{array}{l|l}
I & 0 \\
0 & 0 \\
\hline 0 & I
\end{array}\right), \quad S_{2}=\left(\begin{array}{cc|c}
z_{2} I-A_{2} & 0 \\
I & 0 \\
\hline 0 & I
\end{array}\right), \\
\bar{A}_{11}=A_{1}, \quad \bar{A}_{12}=A_{1} A_{2}+A_{0}, \\
\bar{A}_{21}=I, \quad \bar{A}_{22}=A_{2}, \quad \bar{C}_{2}=C .
\end{gathered}
$$

Proof. Clearly the transformation in (22) is in the required form (10), so it remains to prove the equality and the zero coprimeness of the matrices. In fact, it can be easily verified that the LHS and RHS of (22) both yield the matrix

$$
\left(\begin{array}{cc}
z_{1} z_{2} I-z_{1} A_{2}-z_{2} A_{1}-A_{0} & B \\
0 & 0 \\
-C & 0
\end{array}\right) .
$$

The zero left coprimeness of $\widetilde{P}_{R}$ and $S_{1}$ follows from the fact that the compound matrix

$$
\begin{aligned}
& \left(\begin{array}{ll}
\bar{P}_{R} & S_{1}
\end{array}\right) \\
& \equiv\left(\begin{array}{ccccc}
z_{1} I-A_{1} & -A_{1} A_{2}-A_{0} & B & I & 0 \\
-I & z_{2} I-A_{2} & 0 & 0 & 0 \\
0 & -C & 0 & 0 & I
\end{array}\right)
\end{aligned}
$$


has the highest-order minor which is equal to 1, obtained by deleting the block columns 2 and 3. Similarly the zero right coprimeness of $P_{\mathrm{FM} 1}$ and $S_{2}$ follows from the fact that the compound matrix

$$
\left(\begin{array}{c}
P_{\mathrm{FM} 1} \\
S_{2}
\end{array}\right) \equiv\left(\begin{array}{cc}
z_{1} z_{2} I-z_{1} A_{2}-z_{2} A_{1}-A_{0} & B \\
-C & 0 \\
\hline z_{2} I-A_{2} & 0 \\
I & 0 \\
0 & I
\end{array}\right)
$$

has the highest-order minor which is equal to 1 , obtained from the last two block rows.

\section{Equivalence of Singular 2D Linear Models}

One of the limitations of the regular 2D models is they can be used only to describe $2 \mathrm{D}$ proper transfer functions. In other words, they are suitable only for the representation of northeast quarter plane $2 \mathrm{D}$ systems. To overcome this problem of causality, several versions of these models have been proposed by Kaczorek [14, 20] and have been shown in [21] to be equivalent in the sense that they can all be embedded in the 2D singular general model. In the following, the PMDs associated with the various singular models are considered and the exact form of the transformation linking them is again established.

The singular 2D general model (GM) is given by Kaczorek [14]:

$$
\begin{aligned}
\operatorname{Ex}(i+1, j+1)= & A_{2} x(i+1, j)+A_{1} x(i, j+1) \\
& +A_{0} x(i, j)+B_{2} u(i+1, j) \\
& +B_{1} u(i, j+1)+B_{0} u(i, j) \\
y(i, j)= & C x(i, j)+D u(i, j),
\end{aligned}
$$

where $E, A_{i}, B_{i}, C, D$ are real matrices of appropriate dimensions and $E$ is singular. Then taking the $2 \mathrm{D} z$-transform of (28) and assuming zero boundary conditions yield the PMD:

$$
\begin{aligned}
& P_{\mathrm{GM}} \\
& \quad=\left(\begin{array}{c|c}
z_{1} z_{2} E-z_{1} A_{2}-z_{2} A_{1}-A_{0} & z_{1} B_{2}+z_{2} B_{1}+B_{0} \\
\hline-C & D
\end{array}\right) .
\end{aligned}
$$

The matrix description in (29) describes a number of singular 2D linear models as special cases. In particular setting $B_{1}=B_{2}=0$ in (29) gives rise to the matrix description

$$
P_{\mathrm{SFM} 1}=\left(\begin{array}{c|c}
z_{1} z_{2} E-z_{1} A_{2}-z_{2} A_{1}-A_{0} & B_{0} \\
\hline-C & D
\end{array}\right)
$$

which is associated with the first singular Fornasini-Marche sini model (SFM1). Alternatively when $B_{0}=0$ in (29), the resulting matrix description is that of the second singular Fornasini-Marchesini model (SFM2),

$$
P_{\mathrm{SFM} 2}=\left(\begin{array}{c|c}
z_{1} z_{2} E-z_{1} A_{2}-z_{2} A_{1}-A_{0} & z_{1} B_{2}+z_{2} B_{1} \\
\hline-C & D
\end{array}\right) .
$$

The matrix description associated with the singular Attasi model (SA) is obtained from (30) by setting $A_{0}=-A_{1} A_{2}=$ $-A_{2} A_{1}$, that is,

$$
\begin{aligned}
& P_{\mathrm{SA}}\left(z_{1}, z_{2}\right) \\
& \quad=\left(\begin{array}{c|c}
z_{1} z_{2} E-z_{1} A_{2}-z_{2} A_{1}+A_{1} A_{2} & z_{1} B_{2}+z_{2} B_{1}+B_{0} \\
\hline-C & D
\end{array}\right) .
\end{aligned}
$$

A different type of singular model is the singular Roessertype model (SR):

$$
\begin{gathered}
E\left(\begin{array}{l}
x_{1}(i+1, j) \\
x_{2}(i, j+1)
\end{array}\right)=\left(\begin{array}{ll}
A_{11} & A_{12} \\
A_{21} & A_{22}
\end{array}\right)\left(\begin{array}{l}
x_{1}(i, j) \\
x_{2}(i, j)
\end{array}\right)+\left(\begin{array}{l}
B_{1} \\
B_{2}
\end{array}\right) u(i, j) \\
y(i, j)=\left(\begin{array}{ll}
C_{1} & C_{2}
\end{array}\right)\left(\begin{array}{l}
x_{1}(i, j) \\
x_{2}(i, j)
\end{array}\right)+D u(i, j),
\end{gathered}
$$

where the matrix $E$ is singular. The matrix description arising from (33) is of the form

$$
P_{\mathrm{SR}}=\left(\begin{array}{c|c}
z_{1} E_{2}+z_{2} E_{1}-A & B \\
\hline-C & D
\end{array}\right)
$$

In the following we establish the exact form of the connection between the PMD in the singular general form (29) and the corresponding singular Roesser form in (34). We will show that the transformation involved is that of F-SSE. The connections between all other singular models and the singular Roesser model are deduced as special cases.

Theorem 8. Given an arbitrary $(r+m) \times(r+n)$ singular $2 D$ general matrix description $P_{\mathrm{GM}}$ in the form (29), then $P_{\mathrm{GM}}$ is F-SSE to a singular Roesser matrix description $\bar{P}_{\mathrm{SR}}$ in the form (34). That is,

$$
S_{1} P_{\mathrm{GM}}=\bar{P}_{\mathrm{SR}} S_{2}
$$

where

$$
\begin{gathered}
\bar{P}_{\mathrm{SR}} \\
=\left(\begin{array}{ccccc}
I & -z_{1} I & 0 & 0 & 0 \\
z_{2} E-A_{1} & -z_{2} A_{2}-A_{0} & z_{1} B_{2}+z_{2} B_{1}+B_{0} & 0 & 0 \\
0 & -C & D & I_{m} & 0 \\
0 & 0 & -I_{n} & 0 & I_{n} \\
\hline 0 & 0 & 0 & -I_{m} & 0
\end{array}\right) .
\end{gathered}
$$


The matrices $\bar{E}_{1}, \bar{E}_{2}, \bar{A}, \bar{B}, \bar{C}, \bar{D}$ corresponding to (34) are obtained from the matrices $E, A_{i}, B_{i}, C, D$ given in (29).

$$
S_{1}=\left(\begin{array}{cc}
0 & 0 \\
I & 0 \\
0 & 0 \\
0 & 0 \\
\hline 0 & I_{m}
\end{array}\right), \quad S_{2}=\left(\begin{array}{cc}
z_{1} I & 0 \\
I & 0 \\
0 & I_{n} \\
C & -D \\
\hline 0 & I_{n}
\end{array}\right)
$$

Proof. Consider the following system transformation:

$$
S_{1} P_{\mathrm{GM}}=\bar{P}_{\mathrm{SR}} S_{2},
$$

where

$$
S_{1}=\left(\begin{array}{cc}
0 & 0 \\
I_{r} & 0 \\
0 & 0 \\
0 & 0 \\
\hline 0 & I_{m}
\end{array}\right), \quad S_{2}=\left(\begin{array}{cc}
z_{1} I_{r} & 0 \\
I_{r} & 0 \\
0 & I_{n} \\
C & -D \\
\hline 0 & I_{n}
\end{array}\right)
$$

and the resulting singular Roesser-type system matrix $\bar{P}_{\mathrm{SR}}$ is given by

$$
\left(\begin{array}{ccccc}
I_{r} & -z_{1} I_{r} & 0 & 0 & 0 \\
z_{2} E-A_{1} & -z_{2} A_{2}-A_{0} & z_{1} B_{2}+z_{2} B_{1}+B_{0} & 0 & 0 \\
0 & -C & D & I_{m} & 0 \\
0 & 0 & -I_{n} & 0 & I_{n} \\
\hline 0 & 0 & 0 & -I_{m} & 0
\end{array}\right)
$$

The matrices $\bar{E}_{1}, \bar{E}_{2}, \bar{A}, \bar{B}, \bar{C}$, and $\bar{D}$ corresponding to (34) are

$$
\begin{gathered}
\bar{E}_{1}=\left(\begin{array}{cccc}
0 & 0 & 0 & 0 \\
E & -A_{2} & B_{1} & 0 \\
0 & 0 & 0 & 0 \\
0 & 0 & 0 & 0
\end{array}\right), \quad \bar{E}_{2}=\left(\begin{array}{cccc}
0 & -I_{r} & 0 & 0 \\
0 & 0 & B_{2} & 0 \\
0 & 0 & 0 & 0 \\
0 & 0 & 0 & 0
\end{array}\right), \\
\bar{A}=\left(\begin{array}{cccc}
-I_{r} & 0 & 0 & 0 \\
A_{1} & A_{0} & -B_{0} & 0 \\
0 & C & -D & -I_{m} \\
0 & 0 & I_{n} & 0
\end{array}\right), \\
\bar{B}=\left(\begin{array}{llll}
0 & 0 & 0 & I_{n}
\end{array}\right)^{T}, \quad \bar{C}=\left(\begin{array}{llll}
0 & 0 & 0 & I_{m}
\end{array}\right), \quad \bar{D}=0 .
\end{gathered}
$$

Clearly the transformation in (38) is in the required form (10), so it remains to prove the equality and the zero coprimeness of the matrices. In fact, it can be easily verified that the LHS and RHS of (38) both yield the matrix

$$
\left(\begin{array}{cc}
0 & 0 \\
z_{1} z_{2} E-z_{1} A_{2}-z_{2} A_{1}-A_{0} & z_{1} B_{2}+z_{2} B_{1}+B_{0} \\
0 & 0 \\
0 & 0 \\
C & -D
\end{array}\right) .
$$

The zero left coprimeness of $P_{\mathrm{SR}}$ and $S_{1}$ follows from the fact that the matrix

$$
\left(\begin{array}{ll}
P_{\mathrm{SR}} & S_{1}
\end{array}\right)
$$

has the highest-order minor

$$
\left|\begin{array}{ccccc}
I_{r} & 0 & 0 & 0 & 0 \\
z_{2} E-A_{1} & 0 & 0 & I_{r} & 0 \\
0 & I_{m} & 0 & 0 & 0 \\
0 & 0 & I_{n} & 0 & 0 \\
0 & -I_{m} & 0 & 0 & I_{m}
\end{array}\right|=1
$$

obtained by deleting the second and third block columns. Similarly the zero right coprimeness of $P_{\mathrm{GM}}$ and $S_{2}$ follows from the fact that the matrix

$$
\left(\begin{array}{c}
P_{\mathrm{GM}} \\
S_{2}
\end{array}\right)
$$

has the highest-order minor

$$
\left|\begin{array}{cc}
I_{r} & 0 \\
0 & I_{n}
\end{array}\right|=1
$$

obtained by deleting all the block rows except the 4th and 5th.

\section{Conclusions}

The paper established the connection between the PMDs associated with the various regular and singular 2D linear discrete models. The transformation of strict system equivalence in the sense of Fuhrmann turned out to provide such connection. The results show the relevance of this transformation in another area of 2D polynomial systems theory.

\section{Acknowledgment}

The author wishes to express his thanks to the Sultan Qaboos University (Oman) for their support in carrying out this research work.

\section{References}

[1] H. H. Rosenbrock, State Space and Multivariable Theory, Nelson-Wiley, London, UK, 1970.

[2] P. A. Fuhrmann, "On strict system equivalence and similarity," International Journal of Control, vol. 25, no. 1, pp. 5-10, 1977.

[3] D.S. Johnson, Coprimeness in multidimensional system theory and symbolic computation, Ph.D. thesis, Loughborough University of Technology, Leicestershire, UK, 1993.

[4] A. C. Pugh, S. J. McInerney, M. Hou, and G. E. Hayton, "A transformation for 2-D systems and its invariants," in Proceedings of the 35th IEEE Conference on Decision and Control, pp. 2157-2158, Kobe, Japan, December 1996.

[5] A. C. Pugh, S. J. McInerney, M. S. Boudellioua, D. S. Johnson, and G. E. Hayton, "A transformation for 2-D linear systems and a generalization of a theorem of Rosenbrock," International Journal of Control, vol. 71, no. 3, pp. 491-503, 1998.

[6] E. Zerz, "On strict system equivalence for multidimensional systems," International Journal of Control, vol. 73, no. 6, pp. 495-504, 2000.

[7] A. C. Pugh, S. J. McInerney, M. S. Boudellioua, and G. E. Hayton, "Matrix pencil equivalents of a general 2-D polynomial matrix," International Journal of Control, vol. 71, no. 6, pp. 1027-1050, 1998. 
[8] A. C. Pugh, S. J. McInerney, and E. M. O. El-Nabrawy, "Equivalence and reduction of 2-D systems," IEEE Transactions on Circuits and Systems II, vol. 52, no. 5, pp. 271-275, 2005.

[9] M. S. Boudellioua, "An equivalent matrix pencil for bivariate polynomial matrices," International Journal of Applied Mathematics and Computer Science, vol. 16, no. 2, pp. 175-181, 2006.

[10] M. S. Boudellioua, "On the simplification of systems of linear multidimensional equations," in The Sage Days 24 Workshop on Symbolic Computation in Differential Algebra and Special Functions, Hagenberg, Austria, 2010.

[11] R. P. Roesser, "A discrete state-space model for linear image processing," IEEE Transactions on Automatic Control, vol. 20, no. 1, pp. 1-10, 1975.

[12] S. Attasi, "Systemes lineaires a deux indices," Tech. Rep. 31, INRIA, Le Chesnay, France, 1973.

[13] E. Fornasini and G. Marchesini, "State space realization theory of twodimensional filters," IEEE Transactions on Automatic Control, vol. 21, no. 4, pp. 484-492, 1976.

[14] T. Kaczorek, "Singular models of 2-D systems," in Proceedings of the 12th World Congress on Scientific Computation, Paris, France, 1988.

[15] E. Zerz, "Primeness of multivariate polynomial matrices," Systems and Control Letters, vol. 29, no. 3, pp. 139-145, 1996.

[16] E. Zerz, Topics in Multidimensional Linear Systems Theory, Springer, London, UK, 2000.

[17] A. C. Pugh, S. J. McInerney, and E. M. O. El-Nabrawy, "Zero structures of $n$-D systems," International Journal of Control, vol. 78, no. 4, pp. 277-285, 2005.

[18] E. Fornasini and G. Marchesini, "Doubly-indexed dynamical systems: State-space models and structural properties," Mathematical Systems Theory, vol. 12, no. 1, pp. 59-72, 1978.

[19] M. Morf, B. C. Levy, and S. Y. Kung, "New results in 2-D systems theory: part II: state-space models—realization and the notions of controllability, observability, and minimality," Proceedings of the IEEE, vol. 65, no. 6, pp. 945-961, 1977.

[20] T. Kaczorek, Two-Dimensional Linear Systems, Springer, London, UK, 1985.

[21] T. Kaczorek, "Equivalence of singular 2-D linear models," Bulletin of the Polish Academy of Sciences-Technical Sciences, vol. 37, no. 5-6, pp. 263-267, 1989. 

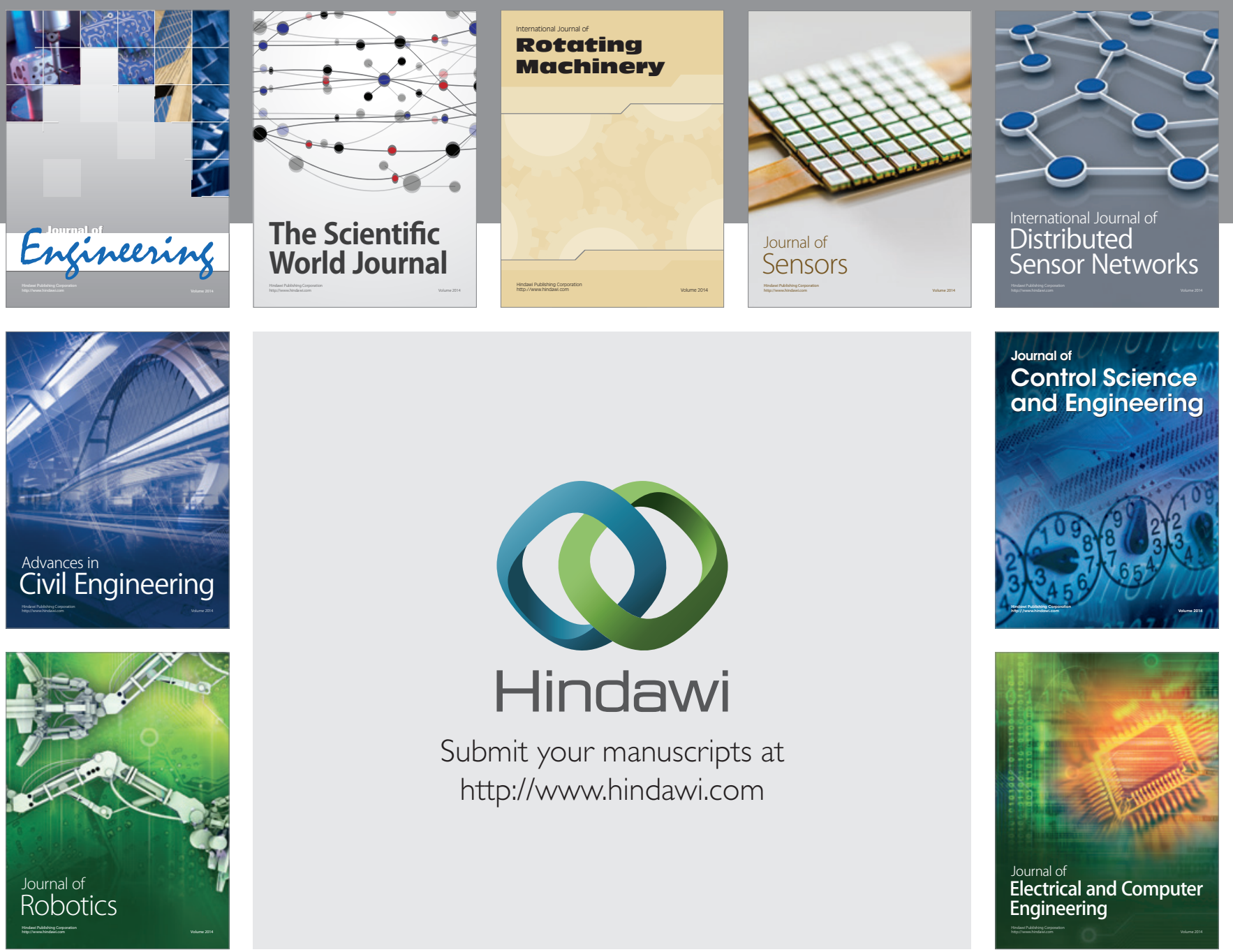

Submit your manuscripts at

http://www.hindawi.com
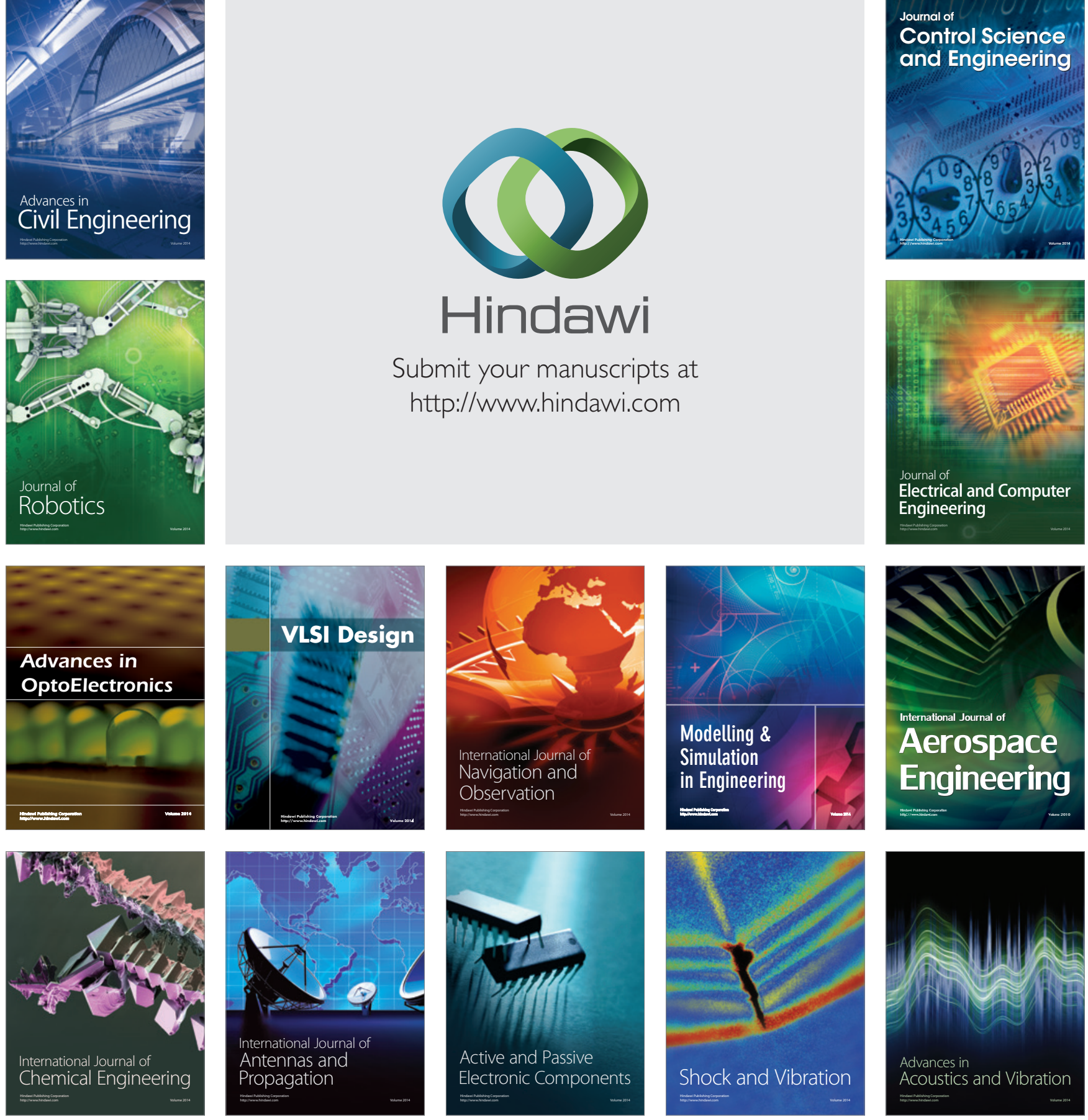